\title{
Low cost patterning of poly(3,4-ethylenedioxythiophene) poly(styrenesulfonate) films to increase organic photovoltaic device efficiency
}

\author{
J. B. Emah, R. J. Curry, ${ }^{\text {a) }}$ and S. R. P. Silva \\ Nanoelectronics Centre, Advanced Technology Institute, University of Surrey, Guildford GU2 7XH, United \\ Kingdom
}

(Received 15 February 2008; accepted 22 July 2008; published online 8 September 2008)

\begin{abstract}
A low-cost lithographic technique to pattern poly(3,4-ethylenedioxythiophene) poly(styrenesulfonate) (PEDOT:PSS) films with $10 \mathrm{~nm}$ deep features of $700 \mathrm{~nm}$ periodicity is demonstrated. The use of these patterned films in poly(3-hexylthiophene): $[6,6]$-phenylC ${ }_{61}$-butyric acid methyl ester organic photovoltaic devices leads to an increase in short circuit current $\left(J_{\mathrm{sc}}\right)$, fill factor, and power conversion efficiency (PCE) with only a slight reduction in open circuit voltage. Patterning the PEDOT:PSS at $150{ }^{\circ} \mathrm{C}$ increases $J_{\mathrm{sc}}$ from 2.44 to $3.03 \mathrm{~mA} / \mathrm{cm}^{2}$ improving the PCE from $0.63 \%$ to $0.81 \%$ with similar increases due to patterning also being obtained at other temperatures. (ㅇ 2008 American Institute of Physics. [DOI: 10.1063/1.2973342]
\end{abstract}

Application of organic conjugated materials in photovoltaic (PV) devices is currently a vast field of interest to researchers. ${ }^{1,2}$ Attractive features include the possibility of solution processing, compatibility with flexible substrates, and low-cost large-scale industrial production, all of which offer the prospect of cheaper PV energy generation. Improvements in device design has led to power conversion efficiencies (PCEs) between $4.5 \%$ and $5 \%$ being reported, ${ }^{3-5}$ which still remain comparatively low when compared to $\mathrm{Si}$ based devices for example. A doubling of this efficiency is required for widespread usage of organic PVs.

The relatively low charge mobility of most organic materials limits the typical film thickness used in devices to less than ideal for efficient absorption of the solar spectrum. ${ }^{6} \mathrm{Im}$ proving charge collection efficiency would therefore allow the use of thicker films resulting in an additional gain due to increased absorption.

In this letter, we examine patterning of a poly $(3,4-$ ethylenedioxythiophene) poly(styrenesulfonate) (PEDOT:PSS) film to improve the charge collection efficiency in blended poly(3-hexylthiophene) (P3HT) and $[6,6]$-phenylC ${ }_{61}$-butyric acid methyl ester (PCBM) based $\mathrm{PV}$ devices. The patterning is undertaken using a low-cost process and is shown to increase the PCE by $30 \%$ in the best of these proof of principle devices, most of which comes in improvement to $J_{\mathrm{sc}}$.

Polydimethylsiloxane (PDMS) (Sylgard 184, Dow Corning) stamps were prepared using a 1:10 ratio of curing agent to base mixed by stirring, followed by degassing under vacuum until the PMDS became clear. The PDMS was then dispensed onto a standard digital versatile disc (DVD) from which the protective polymer coating had been removed to expose the patterned metallic layer. The PDMS film was then heat cured at $70^{\circ} \mathrm{C}$ for $1 \mathrm{~h}$ after which it was allowed to cool before being peeled off the DVD [Fig. 1(a)]. Oxygen plasma treatment $(100 \mathrm{~W}$ for $15 \mathrm{~s})$ of the resulting PDMS stamp was then used to provide a hydrophilic surface.

${ }^{a)}$ Electronic mail: r.j.curry@surrey.ac.uk.
PV devices were fabricated onto cleaned indium tin oxide (ITO) glass substrates $(5-15 \Omega / \square)$ exposed to oxygen plasma (100 W for $10 \mathrm{~min})$. A $45 \pm 5 \mathrm{~nm}$ film of PEDOT:PSS (Sigma Aldrich) was spin coated (3000 rpm) onto the ITO glass and the plasma treated PDMS stamp was placed on top without any pressure other than the weight of the stamp being applied. The PEDOT:PSS was then annealed at the desired temperature (vide infra) for $30 \mathrm{~min}$ under atmospheric conditions following which the PDMS stamp was removed. A $150 \pm 5 \mathrm{~nm}$ film of P3HT:PCBM was spin coated $(600 \mathrm{rpm})$ from a $1: 1 \quad 8 \mathrm{mg} / \mathrm{ml}$ solution of P3HT:PCBM (from Reike Metals and Nano-C respectively) in 1,2-dichlorobenzene. Finally, a $50 \mathrm{~nm}$ aluminum cathode was evaporated through a $2 \mathrm{~mm}$ wide shadow mask yielding three PV devices per substrate. For each set of patterned devices, corresponding reference devices were fabricated which had undergone the same thermal treatment without the PDMS stamp being used.

Surface characterization of the PDMS stamp and patterned PEDOT:PSS surface was performed using a Digital Instruments Dimension 3100 atomic force microscope (AFM) in tapping mode. Current density-voltage $(J-V)$ characteristics were obtained using a Keithley 2400 solar simulator under AM 1.5 illumination.

Figure 1 shows AFM images and the corresponding surface profiles of the PDMS stamp and an imprinted PEDOT:PSS thin film on ITO. The PDMS stamp can be seen to contain regular radial ridgelike features obtained from the DVD master with a height of $\sim 27 \mathrm{~nm}$ and a period of 700 $\mathrm{nm}$. Following stamping and annealing at $150{ }^{\circ} \mathrm{C}$, these features are seen to be transferred to the PEDOT:PSS film. While not as uniform in profile as the stamp, the period remains the same at $700 \mathrm{~nm}$ with a depth of $\sim 10 \mathrm{~nm}$. This demonstrates that the simple procedure described may be used to pattern the polymer film with nanometer scale features.

Figure 2 shows the $J-V$ characteristics of reference and patterned devices fabricated on a $150{ }^{\circ} \mathrm{C}$ annealed nonpatterned and patterned ITO/PEDOT:PSS substrate. Patterned and reference device were fabricated at the same time under 

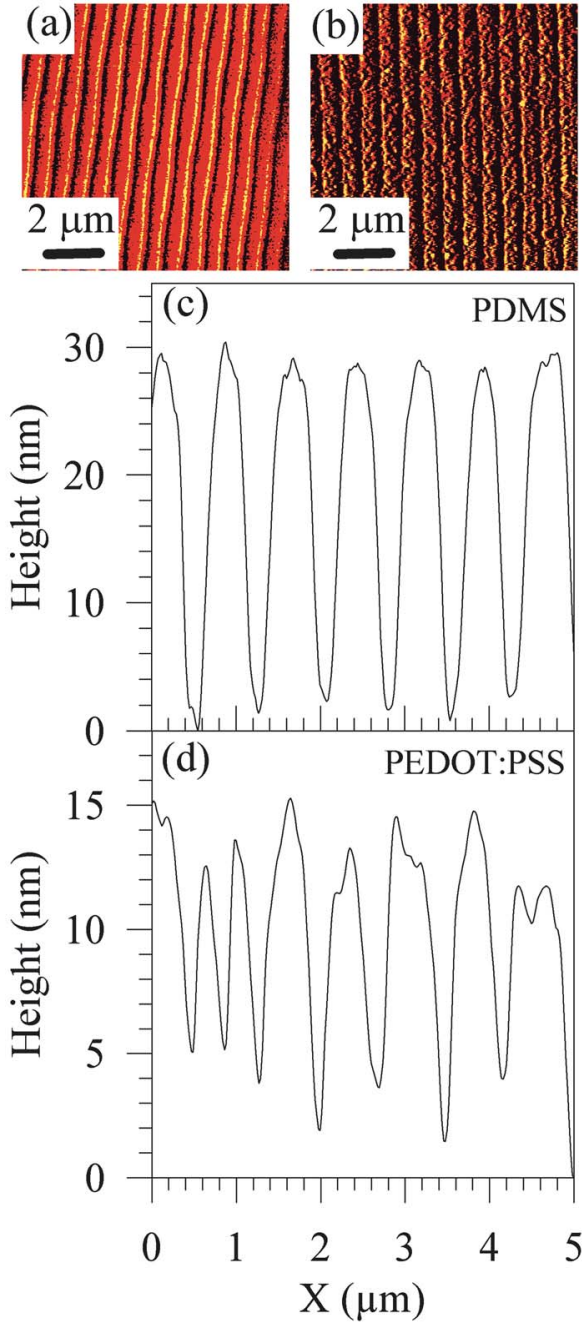

FIG. 1. (Color online) AFM images and corresponding profiles of [(a) and (c)] PDMS stamp (depth $\sim 27 \mathrm{~nm}$, period $\sim 700 \mathrm{~nm}$ ), [(b) and (d)] stamped PEDOT:PSS film annealed at $150{ }^{\circ} \mathrm{C}($ depth $\sim 10 \mathrm{~nm}$, period $\sim 700 \mathrm{~nm})$.

atmospheric conditions, and hence the variation between the patterned and reference device parameters can be used for direct comparison of the effect of patterning. It is clear from Fig. 2 that patterning of the PEDOT:PSS layer leads to a $24 \%$ increase in the short circuit current $\left(J_{\mathrm{sc}}\right)$, from 2.44 to $3.03 \mathrm{~mA} / \mathrm{cm}^{2}$ (see also Table I).

The increase in PEDOT:PSS area by patterning is $~ 3 \%$ (assuming an imprint with sidewall height of $10 \mathrm{~nm}$ every

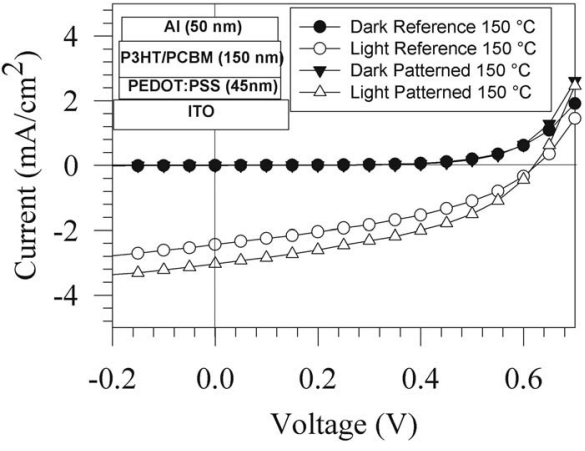

FIG. 2. $J-V$ characteristics of reference and patterned ITO/PEDOT:PSS/ P3HT:PCBM/Al devices under AM 1.5 illumination. The PEDOT:PSS layer was annealed at $150{ }^{\circ} \mathrm{C}$ in both devices with/without a PDMS stamp used to create the patterned/non-patterned surface prior to P3HT:PCBM deposition. Inset shows a schematic of the device structure used.

$700 \mathrm{~nm}$ ), which is significantly less than the increase in $J_{\mathrm{sc}}$. Likewise the maximum increase in volume of P3HT:PCBM provided by the patterning (assuming $150 \mathrm{~nm}$ film remains above the patterns) is only $3.3 \%$. This difference implies an additional effect is present other than a simple increase in electrode area. Possible such effects include (i) improved charge extraction through reduction in PEDOT:PSS thickness, (ii) improved charge extraction through modification of the band alignment at the PEDOT:PSS/P3HT:PCBM interface, and (iii) increased optical absorption due to light trapping or scattering of reflected light by the imprinted pattern. Given that the device fill factor (FF) also increases upon patterning (Table I) along with $J_{\mathrm{sc}}$, this implies that a decrease in series resistance occurs as a result of the patterning. From the data presented in Fig. 1, a reduction in film thickness of $\sim 10 \mathrm{~nm}$ could be present at the "bottom" of the imprinted pattern. This corresponds to a reduction of $\sim 20 \%$ in PEDOT:PSS film thickness at this point and $\sim 10 \%$ overall assuming that the width of the troughs and peaks are similar. However, we note that PEDOT:PSS layer thicknesses ranging from $\sim 50$ to $150 \mathrm{~nm}$ are often used in devices without significant variation of device electrical properties. Modification of the band alignment may occur due to the deposition of small quantities of PDMS on the PEDOT:PSS layer during patterning as reported elsewhere. ${ }^{7}$ We also note that a slight reduction in open circuit voltage $\left(V_{\mathrm{oc}}\right)$ occurs upon patterning, which may also be due to such a modification of the PEDOT:PSS surface. Studies to investigate such

TABLE I. Characteristics of ITO/PEDOT:PSS/PCBM:P3HT/Al PV devices with a patterned and non-patterned (reference) PEDOT:PSS layer. The PEDOT:PSS layer was annealed at the temperature indicated prior to PCBM:P3HT deposition.

\begin{tabular}{cccccc}
\hline \hline Temperature $\left({ }^{\circ} \mathrm{C}\right)$ & Device Type & $J_{\mathrm{sc}}\left(\mathrm{mA} / \mathrm{cm}^{2}\right)$ & $V_{\mathrm{oc}}(\mathrm{V})$ & FF & PCE $(\%)$ \\
\hline 140 & Patterned & 1.58 & 0.55 & 0.47 & 0.41 \\
140 & Reference & 1.52 & 0.61 & 0.29 & 0.27 \\
150 & Patterned & 3.03 & 0.62 & 0.43 & 0.81 \\
150 & Reference & 2.44 & 0.63 & 0.41 & 0.63 \\
160 & Patterned & 1.72 & 0.59 & 0.31 & 0.31 \\
160 & Reference & 1.45 & 0.61 & 0.29 & 0.26 \\
170 & Patterned & 2.01 & 0.56 & 0.41 & 0.46 \\
170 & Reference & 1.20 & 0.63 & 0.29 & 0.22 \\
180 & Patterned & 2.00 & 0.59 & 0.36 & 0.42 \\
180 & Reference & 1.18 & 0.62 & 0.29 & 0.21 \\
\hline \hline
\end{tabular}


possible effects are ongoing and will be reported in due course. In terms of light trapping, any photons reflected from the $\mathrm{Al}$ electrode may be scattered off the features back into the device thus further increasing the effective active layer thickness for absorption.

As a result of the increase in $J_{\mathrm{sc}}$ and FF, the PCE of the device shown in Fig. 2 is also increased from $0.63 \%$ to $0.81 \%$. The electronic parameters that characterize the devices are given in Table I along with those of a number of other devices, similarly fabricated onto ITO/PEDOT:PSS substrates, which had undergone annealing at different temperatures. Each of the values given is the average from measurements on three identical devices. The effect of imprinting can clearly be seen to increase the $J_{\text {sc }}$ in all devices regardless of annealing temperature. Likewise there is an increase in FF as a result of stamping with only a small reduction in $V_{\text {oc }}$ in each case. This leads to an improvement in device efficiency which can be directly related to patterning of the PEDOT:PSS layer. We note that there is some variability in the increase in efficiency as the temperature is changed. Determining the exact cause of this is not possible at present though it could be related to variations in imprinting quality or changes to the PEDOT:PSS conductivity as previously reported. ${ }^{8}$ Alternatively, variations in the definition of the features imprinted could change the strength of any optical confinement or scattering of photons within the device thus increasing absorption.

In summary, we have used a single step soft lithographic technique to pattern the surface of PEDOT:PSS with $\sim 10 \mathrm{~nm}$ deep features with a period of $\sim 700 \mathrm{~nm}$. We demonstrate that through patterning alone the $J_{\mathrm{sc}}, \mathrm{FF}$ and PCE can be increased with only a small reduction in $V_{\mathrm{oc}}$. This is an important step toward further optimization of the organic PV cell performance by patterning the polymer layer between the photoactive layer and the anode.

This work was supported by EPSRC through a Portfolio Partnership award.

${ }^{1}$ N. S. Sariciftci, L. Smilowitz, A. J. Heeger, and F. Wudl, Science 258, 1474 (1992)

${ }^{2}$ C. J. Brabec, N. S. Sariciftci, and J. C. Hummelen, Adv. Funct. Mater. 11, 15 (2001).

${ }^{3}$ W. Ma, C. Yang, X. Gong, K. Lee, and A. J. Heeger, Adv. Funct. Mater. 15, 1617 (2005).

${ }^{4}$ M. Reyes-Reyes, K. Kim, and D. L. Carrol, Appl. Phys. Lett. 87, 083506 (2005)

${ }^{5}$ G. Li, V. Shrotriya, J. Huang, Y. Yao, T. Moriarty, K. Emery, and Y. Yang, Nat. Mater. 4, 864 (2005).

${ }^{6}$ S. Lacic and O. Inganäs, J. Appl. Phys. 97, 124901 (2005).

${ }^{7}$ X. Wang, M. Ostblom, T. Johansson, and O. Inganas, Thin Solid Films 449, 125 (2004).

${ }^{8}$ J. Huang, P. F. Miller, J. S. Wilson, A. J. de Mello, J. C. de Mello, and D. D. C. Bradley, Adv. Funct. Mater. 15, 290 (2005). 